\title{
Solar and Reactor Neutrinos: Upcoming Experiments and Future Projects
}

\author{
Stefan Schönert ${ }^{\text {a }}$ \\ ${ }^{a}$ Max-Planck-Institut für Kernphysik, \\ Saupfercheckweg 1, 69117 Heidelberg, Germany
}

\begin{abstract}
Sub-MeV solar neutrino experiments and long-baseline reactor oscillation experiments toe the cutting edge of neutrino research. The upcoming experiments KamLAND and BOREXINO, currently in their startup and final construction phase respectively, will provide essential information on neutrino properties as well as on solar physics. Future projects, at present under development, will measure the primary solar neutrino fluxes via electron scattering and neutrino capture in real time. High precision data for lepton mixing as well as for stellar evolution theory will become available in the future. This paper aims to give an overview of the upcoming experiments and of the projects under development.
\end{abstract}

\section{Introduction}

In the past, experiments with solar neutrinos and experiments with neutrinos from nuclear reactors probed different ranges of the parameter space for neutrino oscillations. Basically, the distances between source and detector in reactor neutrino experiments $(\lesssim 1 \mathrm{~km})$ were too short to probe the parameter space relevant to explain the solar neutrino deficit [1]:2]. In the very near future, with the upcoming experiments KamLAND [3] and BOREXINO [4], it will be possible to study the parameter space of the large mixing angle (LMA) solution not only with solar, but as well with reactor neutrinos. Although BOREXINO is optimized for solar neutrino detection, it will be able to detect $\bar{\nu}_{e}$ 's from far distant European nuclear reactors [5]. Vice versa, KamLAND is dedicated to reactor neutrino spectroscopy, however, if radio impurities are sufficiently low, it will also measure solar neutrinos.

Future projects, for example LENS [6] and XMASS [7], seek to measure the primary pp-, ${ }^{7} \mathrm{Be}-$ and $\mathrm{CNO}$ neutrino fluxes in real time via neutrino capture and neutrino-electron scattering respectively. Such measurements will provide important information on the flavor of solar neutrinos, on mixing parameters and on solar physics. If the LMA solution is realized in nature, $\Delta m^{2}$ will be determined with high accuracy using reactor neutrinos. However, the measurement of the mixing angle will be statistically limited. Precision measurements of the pp- and ${ }^{7} \mathrm{Be}$ fluxes by elastic scattering (ES) or neutrino capture (CC) would improve considerably the accuracy of the determination of the mixing angle. If the "LOW", "(quasi-) VAC" or small mixing angle (SMA) is the correct solution (see Fig. 1 for terminology), then only solar neutrino experiments can explore the parameter spaces. Full information of the solar neutrino spectrum, via both ES and neutrino capture will be essential in any of the mentioned scenarios.

\section{Objectives of future experiments}

Since flavor conversion has been established from the the combined analysis of SNO and Super-Kamiokande (SK) data [8], the scientific goals of upcoming experiments and future projects shift from discovery and identification of the deficit to a comprehensive study of the phenomena: first, to fix the specific region of the parameter space, and second, to measure with high accuracy the neutrino oscillation parameters and the primary solar neutrino fluxes. 


\subsection{Test of stellar evolution theory}

Solar neutrinos are a unique tool to directly study the fusion processes in the interior of the sun. In particular, direct measurements, with energy information and in real time, of neutrinos from the primary pp-, ${ }^{7} \mathrm{Be}-$ and $\mathrm{CNO}$ processes are of fundamental interest. They will provide complementary information to observational data from helioseismology and probe the predictions of solar models.

\subsection{Neutrino properties}

One of the main goals of future experiments is to study neutrino oscillations and determine the oscillation parameters. In particular they need to:

- confirm $\nu_{e}$ conversion seen by SK and SNO at sub-MeV energies;

- observe directly the signature of oscillations, i.e. time variations or energy spectrum deformations;

- probe flavor composition at sub-MeV energies (active vs. sterile neutrinos, sterile contributions);

- establish the correct oscillation solution (LMA, LOW, (quasi-)VAC, SMA) and measure the mixing parameters with high accuracy.

Other questions comprise: the investigation of $\Theta_{13}$, magnetic moment searches, coherent scattering off nuclei and neutrino instability.

\section{Sub-MeV signature of solar $\nu$ - oscillations}

At ${ }^{8} \mathrm{~B} \nu$-energies the various oscillation solutions differ only slightly with respect to the energy dependence of the survival probability. At sub-MeV energies, however, they differ dramatically. Moreover, for the LOW and VAC solutions the survival probability varies periodically in time for sub-MeV neutrinos. Day/night variations will be observable in the case of the LOW solution and variations on the scale of weeks to months for VAC solutions. Fig. 1 displays the

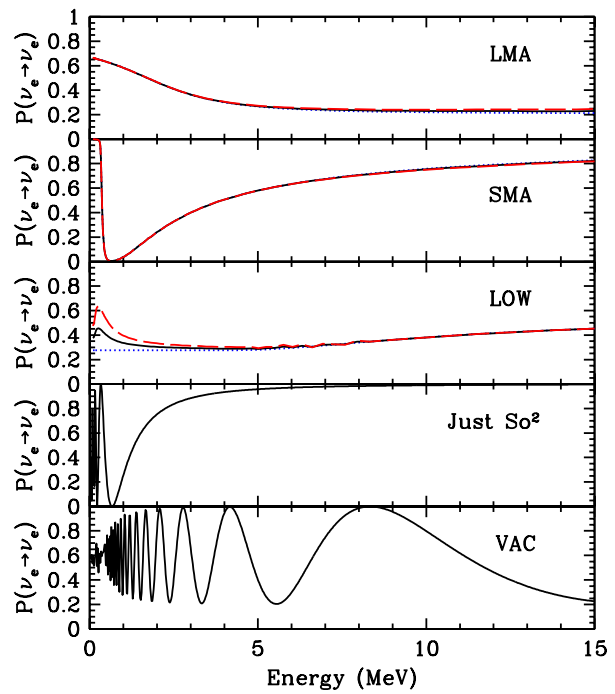

Figure 1. Energy dependent survival probabilities for solar neutrinos for various oscillation scenarios [10]. The characteristic difference of the various solutions at sub-MeV energies should be noted.

characteristic survival curves for various oscillation scenarios. The "Just So" and the "SMA" solutions are disfavored when including the recent results of SNO.

\section{Experiments and R\&D projects}

Table 11 lists sub-MeV solar as well as reactor neutrino experiments which soon become operational, as well as projects which are currently in an early stage of development.

\subsection{ES and CC-detection of sub-MeV neu- trinos}

Two different types of interactions will be exploited for the detection of sub-MeV real-time measurements: $\nu_{x} e$-scattering (ES) which involves charged (CC) and neutral (NC) current interactions, and neutrino capture which only occurs via CC-interaction. The first is sensitive to all active flavors - albeit $\nu_{\mu}, \nu_{\tau}$ interact with a probability of about $1 / 6$ of $\nu_{e}$ - while the latter is sensitive purely to $\nu_{e}$. Combining the results of 
Table 1

Listing of upcoming experiments and projects under development.

\begin{tabular}{|c|c|c|c|c|}
\hline Project & Goals & Method & Technique (Target) & Status \\
\hline \multirow[t]{2}{*}{ KamLAND [3] } & 1) $\bar{\nu}_{e}^{\text {reactor }}$ & $\mathrm{CC}$ & $\mathrm{LS}(\mathrm{CH})$ & $2001 / 2$ \\
\hline & 2) $\nu^{\text {sun }}:{ }^{7} \mathrm{Be},{ }^{8} \mathrm{~B}$ & ES & $\mathrm{LS}(\mathrm{CH})$ & \\
\hline \multirow[t]{2}{*}{ BOREXINO [4] } & 1) $\nu^{\text {sun }}:{ }^{7} \mathrm{Be}$, pep, ${ }^{8} \mathrm{~B}$ & ES & $\mathrm{LS}(\mathrm{CH})$ & $2002 / 3$ \\
\hline & 2) $\bar{\nu}_{e}^{\text {reactor }}$ & $\mathrm{CC}$ & $\mathrm{LS}(\mathrm{CH})$ & $2002 / 3$ \\
\hline LENS [6] & $\nu_{e}^{\text {sun }}: \mathrm{pp},{ }^{7} \mathrm{Be}, \mathrm{CNO}$ & $\mathrm{CC}$ & $\mathrm{LS}(\mathrm{CH}+$ metal $)$ & R\&D: $2002 / 3$ \\
\hline XMASS [7] & $\nu_{x}^{\text {sun }}: \mathrm{pp},{ }^{7} \mathrm{Be}$ & ES & LS (Xe) & R\&D: $2002 / 3$ \\
\hline HERON 12] & $\nu_{x}^{\text {sun }}: \mathrm{pp}$ & ES & LS $(\mathrm{He})$ & $\mathrm{R} \& \mathrm{D}$ \\
\hline CLEAN 『11] & $\nu_{x}^{\text {sun }}: \mathrm{pp}$ & ES & LS $(\mathrm{He}, \mathrm{Ne})$ & R\&D \\
\hline MOON |13n & $\nu_{e}^{s u n}: \mathrm{pp},{ }^{7} \mathrm{Be}$ & $\mathrm{CC}$ & hybrid or LS $(\mathrm{CH}+$ metal $)$ & $\mathrm{R} \& \mathrm{D}$ \\
\hline TPC [14, 15] & $\nu_{x}^{\text {sun }}: \mathrm{pp},{ }^{7} \mathrm{Be}$ & ES & $\mathrm{TPC}(\mathrm{He}+\mathrm{CH})$ & R\&D \\
\hline GENIUS 16 & $\nu_{x}^{\text {sun }}: \mathrm{pp}$ & ES & Germanium & $\mathrm{R} \& \mathrm{D}$ \\
\hline LOW-C14 & $\nu_{x}^{\text {sun }}: \mathrm{pp}$ & ES & $\mathrm{LS}(\mathrm{CH})$ & $R \& D$ \\
\hline Kr2Det 18 & $\bar{\nu}_{e}^{\text {reactor }}: \mathrm{U}_{e 3}$ & $\mathrm{CC}$ & $\mathrm{LS}(\mathrm{CH})$ & R\&D \\
\hline TEXONO 19 & $\bar{\nu}_{e}^{\text {reactor }}: \mu_{\nu}$ & ES & solid scint. & $R \& D$ \\
\hline
\end{tabular}

ES: Elastic scattering of neutrinos off electrons (CC+NC); CC: neutrino capture (charged current; CC); LS: liquid scintillator; $\mathrm{CH}$ : hydro-carbon.

an ES experiment with those of a neutrino capture experiment provides a unique neutral current probe. Currently this provides the only possibility to study the contributions of $\nu_{\mu}$ and $\nu_{\tau}$ in the primary pp- and ${ }^{7}$ Be neutrino fluxes. Electron scattering and neutrino capture experiments are therefore complementary, and should be realized in a coordinated strategy in order to maximize the potential physics output. For purposes of illustration only, Fig. 2 shows the expected relative interaction rates of ${ }^{7} \mathrm{Be}$ neutrinos in BOREXINO and LENS. It should be noted that modest suppression factors, as for example expected for the the LMA solution, challenge the accuracy reachable in the experiments. For instance, in the particular case indicated in Fig. 4.1 with "experimentally difficult", an error of $\lesssim 3 \%$ is needed both for BOREXINO and LENS in order to obtain a signal for $\nu_{\mu}, \nu_{\tau}$ appearance $\gtrsim 3 \sigma \mathrm{CL}$.

\subsection{BOREXINO and KamLAND}

As illustrated in Figs. 3 and 4, the BOREXINO (BX) and KamLAND (KL) detectors are similar in design. BX is optimized for solar neutrino detection, thus design priority has been given to high radiopurity, while KL is optimized for reactor neutrino detection where radiopuri-

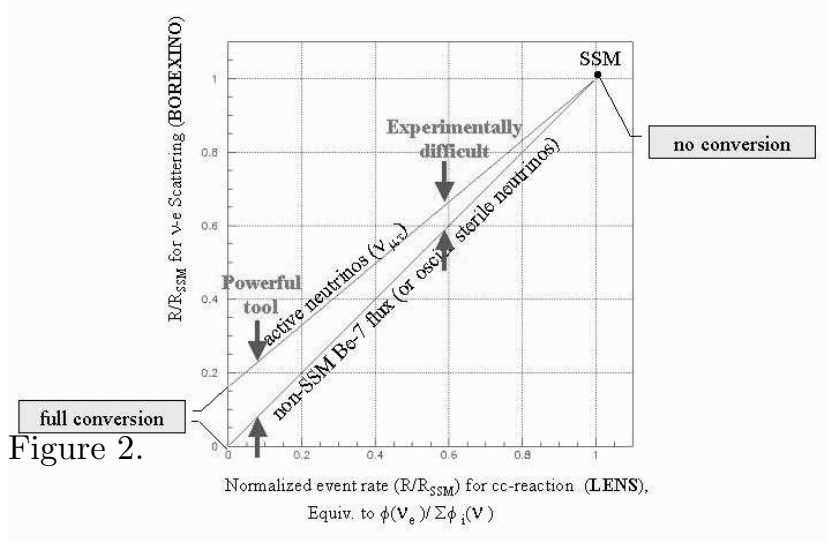

ties are less stringent. Both detectors use liquid scintillators as $\nu$-target and detection medium. Reactor- $\bar{\nu}_{e}$ 's are detected via $\bar{\nu}_{e} p \rightarrow e^{+} n$ where $e^{+}$carries the energy information of the interacting neutrino. The $\bar{\nu}_{e}$-tag is given by a coincident $e^{+}-\mathrm{n}$ signal which is correlated in space and separated in time with $\tau=200 \mu$ sec. Solar neutrinos are measured via $\nu_{x} e^{-}$-scattering $(x=e, \mu, \tau)$. The Compton-like recoil electron provides the energy information of the interaction. About 55 events/day/100 tons are expected in the ${ }^{~}{ }^{7} \mathrm{Be}$ neutrino window" (i.e. recoil electrons with en- 
ergies between $250-800 \mathrm{keV}$ ). Since $\nu_{x} e^{-}$-signals cannot be distinguished from radioactive background as $\beta$-decay events with the same energy, ultra-low backgrounds are required for neutrino detection. The signal and background spectra expected in BOREXINO are displayed in Fig. 5. Expected detector performance and specifications for KL and BX are summarized in Tab. 2. KL needs to meet similar radiopurity specifications to achieve solar- $\nu$ detection. However, variable time effects, such as day/night variations, can be observable even with a background larger than $10^{-16} \mathrm{~g} / \mathrm{g}$. KL data taking commenced the end of 2001 and the first results from reactor $\bar{\nu}_{e^{-}}$ measurements are expected in 2002. BX scintillator filling will be completed early 2003 and first solar neutrino results might be available during the same year.

Table 2

Features of BOREXINO and KamLAND

\begin{tabular}{lll}
\hline & BX & KL \\
\hline Mass: & $300 \mathrm{t}$ & $1 \mathrm{kt}$ \\
LS: & PC+PPO & MO+PC+PPO \\
Yield [pe/MeV]: & 430 & 300 \\
Purities: & & \\
U,Th $[\mathrm{g} / \mathrm{g}]:$ & $\leq 10^{-16^{*}}$ & $\leq 10^{-14^{* *}}$ \\
$\mathrm{~K}[\mathrm{~g} / \mathrm{g}]:$ & $\leq 10^{-14^{*}}$ & - \\
${ }^{14} \mathrm{C} /{ }^{12} \mathrm{C}$ & $\leq 10^{-18}$ & - \\
$\mathrm{CP}:$ & $1 \times \mathrm{GS}$ & $7 \times \mathrm{GS}$ \\
Reactor dist.: & $800 \mathrm{~km}$ & $160 \mathrm{~km}$ \\
\hline
\end{tabular}

LS: Liquid scintillator; PC: pseudocumene; MO: mineral oil; pe: photo electrons; ${ }^{*}$ : for solar- $\nu$ detection, ${ }^{* *}$ : for reactor- $\bar{\nu}_{e}$ 's, CP: cosmogenic production at Kamioka is a factor 7 higher than at Gran Sasso.

The reactor signal in KL (and as well in BX) will be strongly suppressed in the case where LMA parameters drive solar neutrino oscillations. The oscillation length $L[\mathrm{~m}]=1.24$. $E[\mathrm{MeV}] / \Delta m^{2}\left[\mathrm{eV}^{2}\right]$ would amount to $100 \mathrm{~km}$ for $3 \mathrm{MeV}$ reactor- $\bar{\nu}_{e}$ 's and $\Delta m^{2}=3.7 \times 10^{-5} \mathrm{eV}^{2}$. Fig. 6 shows the parameter space in which an observable rate suppression is expected. In case of no oscillations, KL will measure about 800

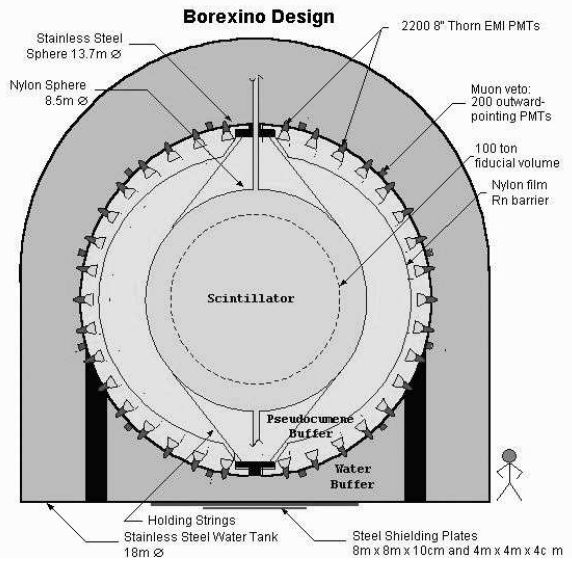

Figure 3. Schematic view of the BOREXINO detector at Gran Sasso, Italy.

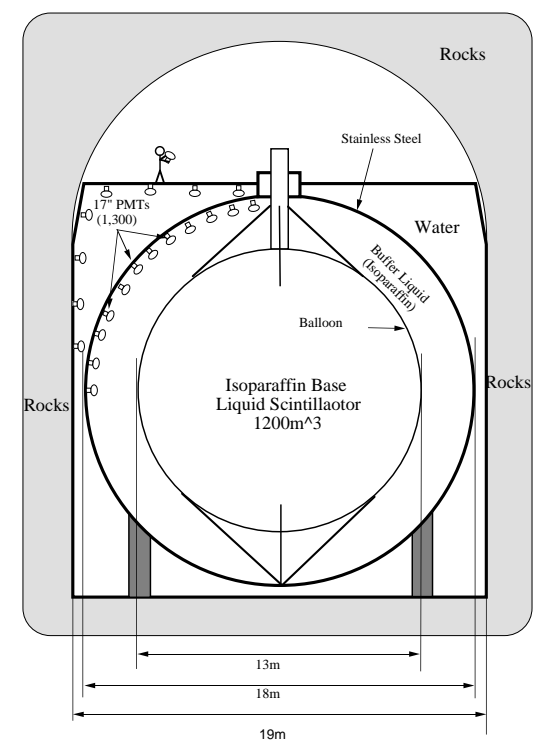

Figure 4. View of the KamLAND detector at Kamioka, Japan. 


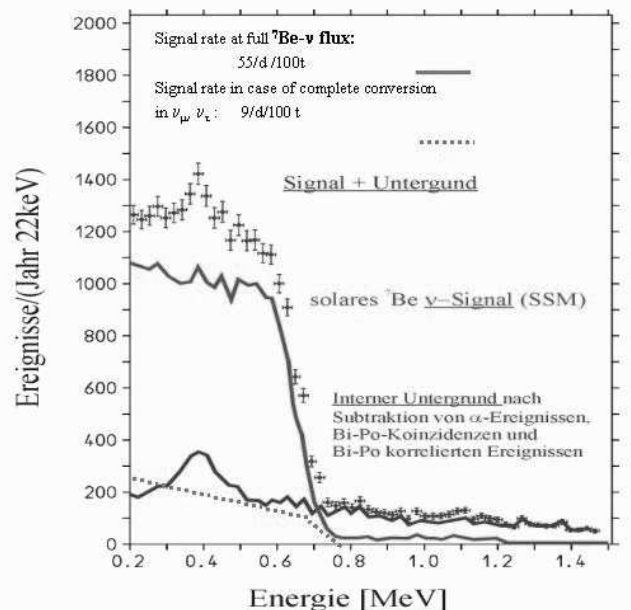

Figure 5. Electron recoil spectrum of ${ }^{7} \mathrm{Be}-\nu$ 's and expected background in BOREXINO.

events per year in comparison to 30 events in BX. This obviously translates to a higher sensitivity with respect to $\sin ^{2} 2 \Theta$. The recent best fit value $\sin ^{2} 2 \Theta=0.79$, however, should provide unambiguous signals in both experiments provided that background levels are as expected.

For $\Delta m^{2}$ values between $\sim 1 \times 10^{-5} \mathrm{eV}^{2}$ and $\sim 2 \times 10^{-4} \mathrm{eV}^{2}$, KL will not only observe a suppression of the rate, but also deformation of the shape of the well known positron energy spectrum. From the shape one can then infer, with high accuracy, the actual value of $\Delta m^{2}$. For values larger $2 \times 10^{-4} \mathrm{eV}^{2}$ the oscillation signal smears out and only a lower limit can be given by KL. The upper "hard core" limit then will be given by the $\mathrm{CHOOZ}$ experiment at around $1 \times 10^{-3} \mathrm{eV}^{2}$. A follow-up experiment would be needed in this particular case. A a reactor experiment with a baseline of about $20 \mathrm{~km}$ is under discussion to address this question [20].

\subsection{ES experiments for sub-MeV $\nu$ detec- tion}

Various $\nu e^{-}$-scattering experiments for pp neutrino detection are under development. With an interaction rate of about $2 /$ ton/day for $\mathrm{pp}-\nu_{e}$, the detectors need to have a target mass of about 10 tons to acquire high statistical accuracy. ${ }^{14} \mathrm{C} /{ }^{12} \mathrm{C}$

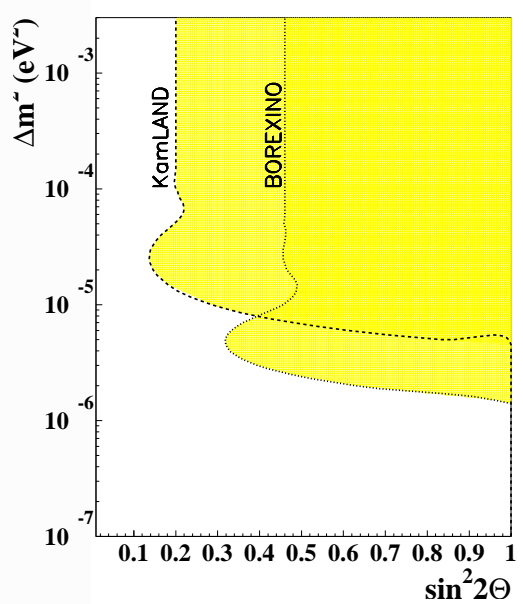

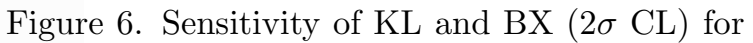
$\nu$-oscillation search with reactor- $\bar{\nu}_{e}$. The shaded area would provide a measurable suppression of the $\bar{\nu}_{e}$-rate based on 3 years of data taking. KL's sensitivity increases to $\sin ^{2} 2 \Theta=0.1$ in the case of background free detection of $\bar{\nu}_{e}$ 's.

ratios at $10^{-18}$ in organic scintillators, as determined with the CTF of BX [21], prohibit the measurement of pp-neutrinos since the $156 \mathrm{keV}$ $\beta$-decay endpoint significantly obscures the ppenergy range. If a low- ${ }^{14} \mathrm{C}$ liquid scintillator with ${ }^{14} \mathrm{C} /{ }^{12} \mathrm{C} \lesssim 10^{-20}$ could be produced, as discussed in 17], a BX type detector could be realized for $\mathrm{pp}-\nu$ detection. A promising approach to overcome the ${ }^{14} \mathrm{C}$ background is to avoid organic liquids and instead, to use liquified noble gas as a scintillator. Projects with helium, neon and xenon are under investigation. Argon and krypton are not suitable because of their long-lived radioactive isotopes. Tab. 3 summarizes features relevant for the design of pp-neutrino detection.

High density liquid xenon, as proposed by the XMASS collaboration [7], provides efficient selfshielding. A geometry similar to that of BX, but smaller in size, could thus be realized. The scintillation photons can be detected with photomultiplier technology at liquid xenon temperatures. Backgrounds can arise from ${ }^{85} \mathrm{Kr} \beta$-decay and from $2 \nu-\beta \beta$ decay of ${ }^{136} \mathrm{Xe}$. The first isotope must be less than $4 \times 10^{-15} \mathrm{~g}$ krypton $/ \mathrm{g}$. If 
Table 3

Characteristics of liquid noble gases as $\nu$-target and detector medium.

\begin{tabular}{llll}
\hline & $\mathrm{He}$ & $\mathrm{Ne}$ & $\mathrm{Xe}$ \\
\hline Boiling Tmp.[K]: & 4.2 & 27 & 165 \\
Z: & 2 & 10 & 54 \\
Density $\left[\mathrm{g} / \mathrm{cm}^{3}\right]:$ & 0.125 & 1.20 & 3.06 \\
Emission wl $[\mathrm{nm}]:$ & 73 & 80 & 175 \\
Photons/MeV: & 22000 & $15000 ?$ & 42000 \\
\hline
\end{tabular}

the $\tau_{1 / 2}$ of ${ }^{136} \mathrm{Xe}$ is $<8 \times 10^{23}$ years, then isotope separation is needed. The best limits have $\tau_{1 / 2}>1.1 \times 10^{22}$ years [22]. Since theoretical calculations estimate $\tau_{1 / 2} \approx 8 \times 10^{21}$ years, the need for isotope separation is likely. Fig. 7 shows the spectrum of recoil electrons along with the $2 \nu-\beta \beta$ decay of ${ }^{136} \mathrm{Xe}$. A prototype detector containing $100 \mathrm{~kg}$ of liquid Xe is under construction. Photon detection will be achieved with newly developed low background photomultiplier tubes consisting of a steel housing and a quartz window. Milestones during this $R \& D$ phase will include the determination of the $2 \nu-\beta \beta$ half-life and the optical properties of liquid xenon, such as the scattering length of scintillation light.

A similar concept is being pursued by the CLEAN project [11]. The target under discussion is neon (or helium) for the fiducial mass and liquid or solid neon as passive shielding buffer. Since neon scintillation occurs at $80 \mathrm{~nm}$, a wavelength shifter is required in order to use photo multipliers for light detection.

About 20t of super-fluid helium as neutrino target is modelled in the HERON project [12]. Recoil electrons are detected via 1) prompt scintillation photons $(80 \mathrm{~nm})$ and 2) delayed phonon/rotons (1 meV; $\left.10^{8} / \mathrm{MeV}\right)$ on sapphire or silicon wafer calorimeters. About 2400 of these wafers are proposed to be located above the liquid phase. A schematic view of the detector is displayed in Fig. 8. The temperature rise after photon/phonon interactions is sensed by super-conducting transition edge thermometers or by magnetic micro-calorimeters. The goal is to obtain single $(16 \mathrm{eV})$ photon sensitivity per wafer. Internal radioactive backgrounds are not

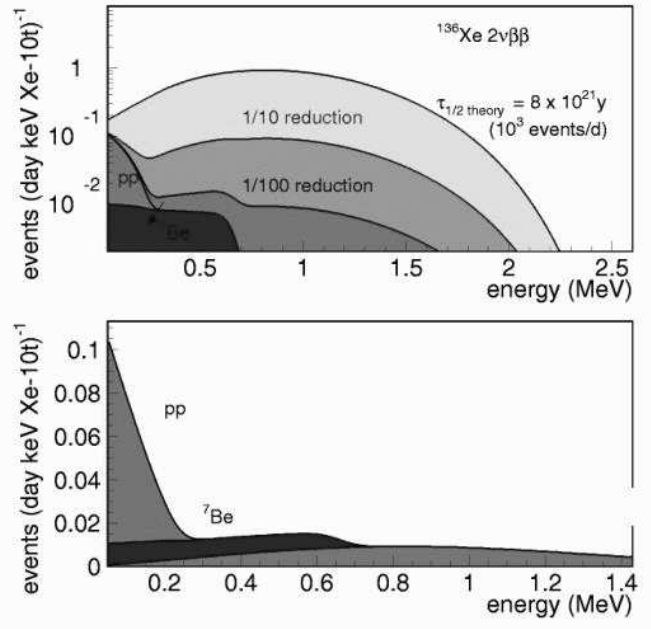

Figure 7. Neutrino recoil spectrum displayed together with the $2 \nu-\beta \beta$-decay of ${ }^{136} \mathrm{Xe}$. Isotope separation is required if $\tau_{1 / 2} \approx 8 \times 10^{21}$ years.

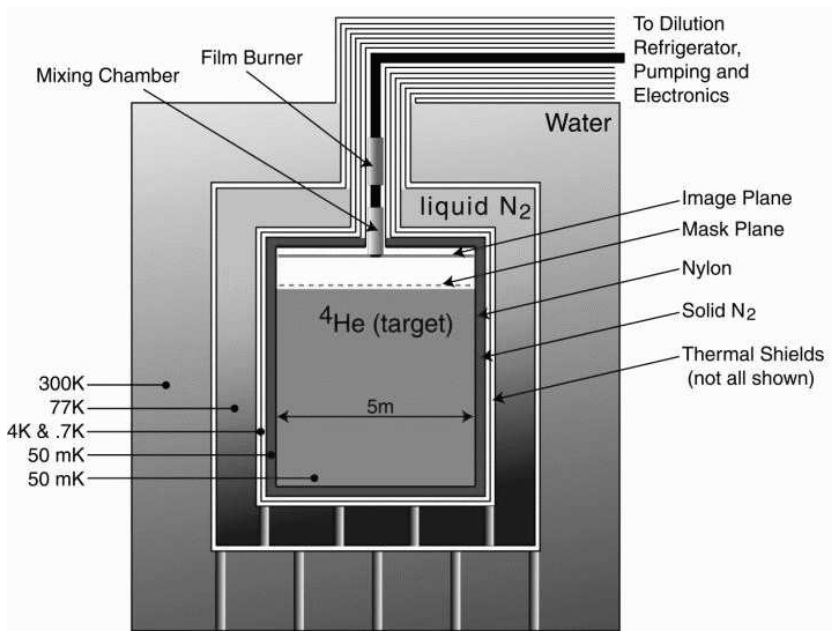

Figure 8. Schematic view of HERON detector. 
expected to be a problem, since super-fluid helium is "self-cleaning" (i.e. gravity $>\mathrm{kT}$ or chemical binding energies) and impurities, if present, fall to the bottom. On the other hand, the low density of helium does not provide sufficient self shielding against external backgrounds. Therefore, high radiopurity shielding material, dewar and light sensors is imperative. Point-like events, such as $\nu e^{-}$-scattering, can possibly be distinguished from multiple scattering events typical for external $\gamma$-radiation by position reconstruction.

Full energy reconstruction of the neutrino energy in ES experiments requires the determination the angle that the recoil electron makes with respect to the incident neutrino direction. For this purpose TPC's for solar neutrino detection are being investigated by several groups. The pioneering research project HELLAZ was one such project. A follow up $\mathrm{R} \& \mathrm{D}$ project was initiated recently 14 that proposes substantial conceptual modifications: it is planned to use methane instead of isobutane to overcome the ${ }^{14} \mathrm{C}$ background, to decrease the operational pressure so as to increase the angular resolution, and to simplify the complexity of the electrodes in order to reduce the radioactive background. A further project under discussion is Super-MUNU 15]. The detector concept is based on the experience of the MUNU experiment that searched for a neutrino magnetic moment at the Bugey reactor. Four $50 \mathrm{~m}^{3}$ modules, filled with $\mathrm{CF}_{4}$ at 1-2 bars pressure are under discussion. As a prototype study, it has been proposed to operate the MUNU reactor detector in an underground laboratory in order to study radioactive background and detector performance (angular and energy resolution) at $\mathrm{pp}-\nu$ energies.

\subsection{Charged current experiments for sub- $\mathrm{MeV} \nu_{e}$ real time detection}

The electron flavor content of sub-MeV solar neutrinos will be probed via neutrino capture. Only a few promising candidate nuclei exist for real time detection. Either the transition must populate an isomeric excited state, or the final state needs to be unstable in order to provide a coincidence tag to discriminate against back-

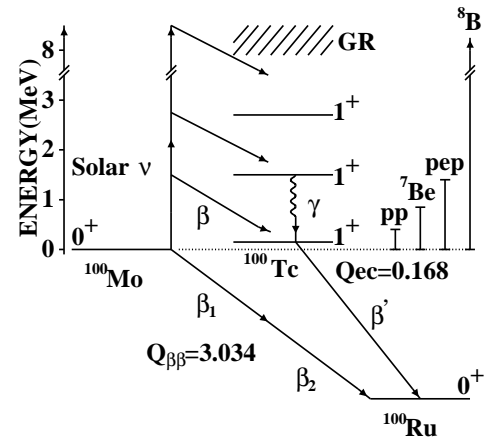

Figure 9. Level and transition scheme of ${ }^{100} \mathrm{Mo}$ for solar neutrino induced $\beta \beta^{\prime}$ decay [13].

ground events. Moreover, the energy threshold of the transition needs to be sufficiently low to have sensitivity to sub-MeV neutrinos. Tab. 1 lists nuclei which satisfy these condition.

The MOON collaboration 13] favors the molybdenum isotope ${ }^{100} \mathrm{Mo}$ for solar neutrino detection. The threshold for neutrino capture is 168 $\mathrm{keV}$ and thus sensitive to pp-neutrinos. The GT strength to the $1^{+}$ground state in ${ }^{100} \mathrm{Tc}$ is measured to be $\left(g_{A} / g_{V}\right)^{2} B(G T)=0.52 \pm 0.02$ by EC and charge exchange reactions. An interaction rate of 1.1 and 3.3 events per day and 10 tons of ${ }^{100} \mathrm{Mo}$ is expected for pp- and ${ }^{7} \mathrm{Be}$ neutrinos. Fig. 9 displays the nuclear levels and transition involved in the neutrino capture process. The neutrino signature consists of a prompt electron and a delayed $\beta$-decay $(\tau=15.8 \mathrm{~s})$ with endpoint energy of $3.4 \mathrm{MeV}$. Two different design options for a detector based on 100 tons of natural molybdenum (9.6 tons of ${ }^{100} \mathrm{Mo}$ ) exist: 1) a hybrid detector consisting of plastic scintillator bars interleaved by Mo-foils $\left(0.05 \mathrm{~g} / \mathrm{cm}^{2}\right)$ and read out with PMTs via wave-length shifting fibers, or 2) a similar detector geometry with liquid scintillators instead of plastic. Fig. 10 shows the expected spectrum together with the background of $2 \nu-\beta \beta$ decay.

Various candidate nuclei listed in Tab. 1 are being investigated by the LENS collaboration [6]. These metals are being loaded up to $10 \%$ 
Table 4

Candidate nuclei for real time solar neutrino detection via neutrino capture (CC).

\begin{tabular}{lllllll}
\hline Isotope & Abundance & $\mathrm{T}_{1 / 2}$ & Daughter & $\mathrm{Q}[\mathrm{keV}]$ & $\mathrm{E}^{*}[\mathrm{keV}]$ & $\mathrm{T}_{1 / 2}^{*}$ \\
\hline${ }^{115} \mathrm{In}$ & $95.7 \%$ & $5 \cdot 10^{14} \mathrm{a}$ & ${ }^{115} \mathrm{Sn}$ & 128 & $116+498$ & $3.26 \mu \mathrm{s}$ \\
${ }^{176} \mathrm{Yb}$ & $12.7 \%$ & stable & ${ }^{176} \mathrm{Lu}$ & 301 & 72 & $50 \mathrm{~ns}$ \\
${ }^{160} \mathrm{Gd}$ & $21.9 \%$ & stable & ${ }^{160} \mathrm{~Tb}$ & 244 & $75+64$ & $6+60 \mathrm{~ns}$ \\
${ }^{82} \mathrm{Se}$ & $9.4 \%$ & $1 \cdot 10^{20} \mathrm{a}$ & ${ }^{82} \mathrm{Br}$ & 173 & 29 & $10 \mathrm{~ns}$ \\
${ }^{100} \mathrm{Mo}$ & $9.6 \%$ & stable & ${ }^{100} \mathrm{Tc}$ & 168 & $3209(\beta$-decay of ground state) & $15.8 \mathrm{~s}$ \\
${ }^{71} \mathrm{Ga}$ & $39.9 \%$ & stable & ${ }^{71} \mathrm{Ge}$ & 404 & 175 & $79 \mathrm{~ns}$ \\
${ }^{137} \mathrm{Ba}$ & $11.2 \%$ & stable & ${ }^{137} \mathrm{La}$ & 611 & 11 & $89 \mathrm{~ns}$ \\
${ }^{123} \mathrm{Sb}$ & $42.7 \%$ & stable & ${ }^{123} \mathrm{Te}$ & 541 & $330+159$ & $31 \mathrm{~ns}$ \\
${ }^{159} \mathrm{~Tb}$ & $100 \%$ & stable & ${ }^{159} \mathrm{Dy}$ & 543 & $177+121+56$ & $9.3 \mathrm{~ns}$ \\
\hline
\end{tabular}

$\mathrm{E}^{*}$ and $\mathrm{T}_{1 / 2}^{*}$ refer to de-excitation energy and half life of the isomeric state populate in the transition.
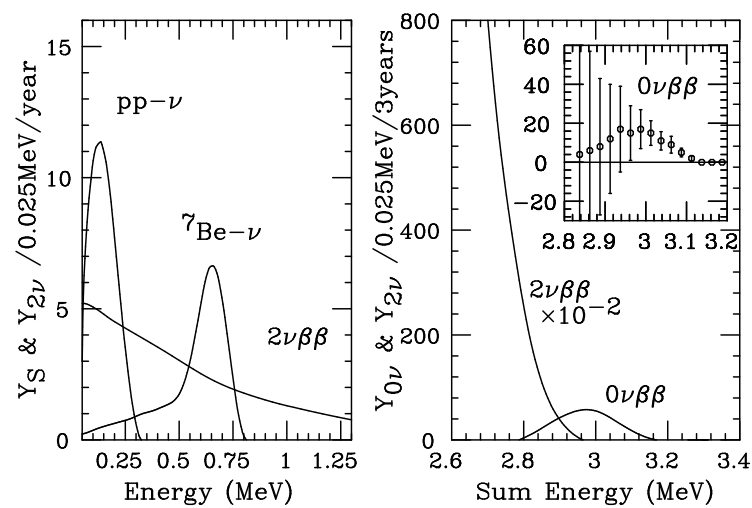

Figure 10. Energy spectrum for a possible detector with 3.3 tons of ${ }^{100} \mathrm{Mo}$. The continuous line shows the expected background from $2-\nu \beta \beta$ decay $[13]$.

in weight in organic liquid scintillators. Ligands under study are carboxylic acids, phosphor organic compounds as well as beta-diketonates. Among the various nuclei, ${ }^{115} \mathrm{In}$ and ${ }^{176} \mathrm{Yb}$ are currently favored. Figs. 11 and 12 display the levels and transitions involved. ${ }^{82} \mathrm{Se}$ and ${ }^{160} \mathrm{Gd}$ are less promising because of challenges related to detection technique as well as to radioactive background.

The $B(G T)$ transition strengths of ${ }^{176} \mathrm{Yb}$ and ${ }^{115}$ In have been measured via charge exchange reactions in order to estimate the interaction cross section. For ${ }^{176} \mathrm{Yb}$ a $B(G T)$ value of $0.20 \pm 0.04$ $(0.11 \pm 0.02)$ was evaluated for the transition to

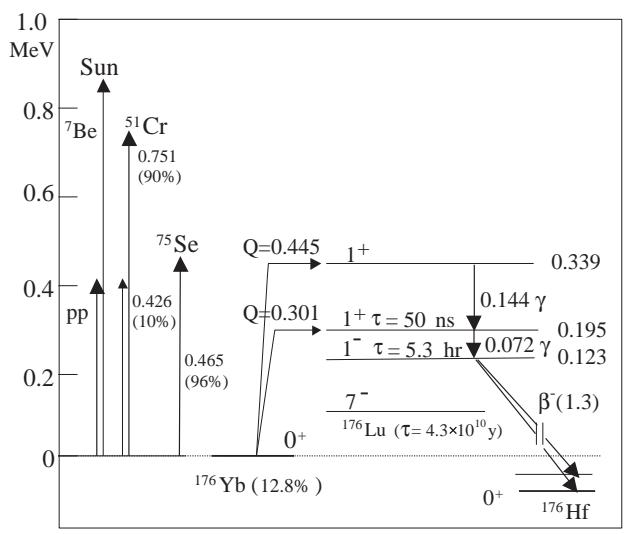

Figure 11. Level scheme and $\gamma$-ray tag for solar neutrino detection by ${ }^{176} \mathrm{Yb}$. All energies in $\mathrm{MeV}$ 23.

the $194.5 \mathrm{keV}(339 \mathrm{keV})$ level [23]. For ${ }^{115} \mathrm{In}$ the weak matrix element is $B(G T)=0.17$ [24]. These values are sufficiently accurate to evaluate the target mass necessary for the LENS detector. However, to derive the neutrino fluxes, it is planned to use an artificial ${ }^{51} \mathrm{Cr}$ neutrino source of several MCi strength to calibrate the cross section at the few percent level. Figs. 13 and 14 display the expected spectra and interaction rates for $20 \mathrm{t}$ of natural ytterbium and $4 \mathrm{t}$ of indium. The actual target mass for an indium detector will most likely be on the $10 \mathrm{t}$ scale taking into account detector efficiencies and required statistical accuracy. 


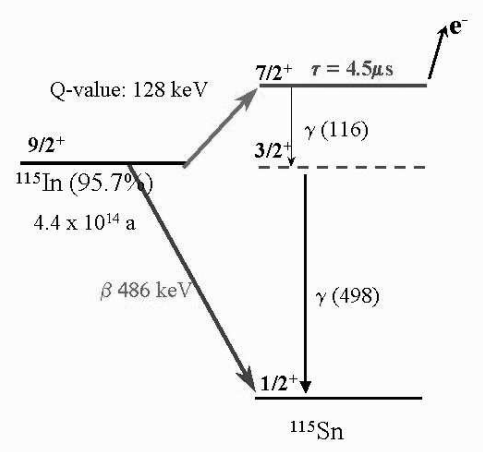

Figure 12. Level scheme and $\gamma$-ray tag for solar neutrino detection by ${ }^{115} \mathrm{In}$.

A high granularity is required in order to discriminate against background caused by random coincidences of ${ }^{14} \mathrm{C}$ and other sources. This will be realized by a modular detector design. A basic module has a lengths of the order of the absorption length of the liquid scintillator which corresponds to a few meters. The modul area varies from $5 \times 5 \mathrm{~cm}^{2}$ to $20 \times 20 \mathrm{~cm}^{2}$ depending on metal loading, scintillator performance, choice of nucleus and neutrino source calibration optimization.

In order to study the detector performance as close as possible to the final detector geometry, the LENS Low-Background-Facility (LLBF) has been newly installed underground at the LNGS. A low-background passive shielding system with 80 tons of mass, located in a clean room, can house detector modules with dimensions up to $70 \mathrm{~cm} \times 70 \mathrm{~cm} \times 400 \mathrm{~cm}$. All shielding materials have been selected in order to minimize the intrinsic radioactive contamination. First results from the prototype phase are expected in 2002. Of particular interest will be the experimental study whether pp- $\nu_{e}$ detection with ${ }^{115} \mathrm{In}$, as proposed in Ref. [25] is feasible.

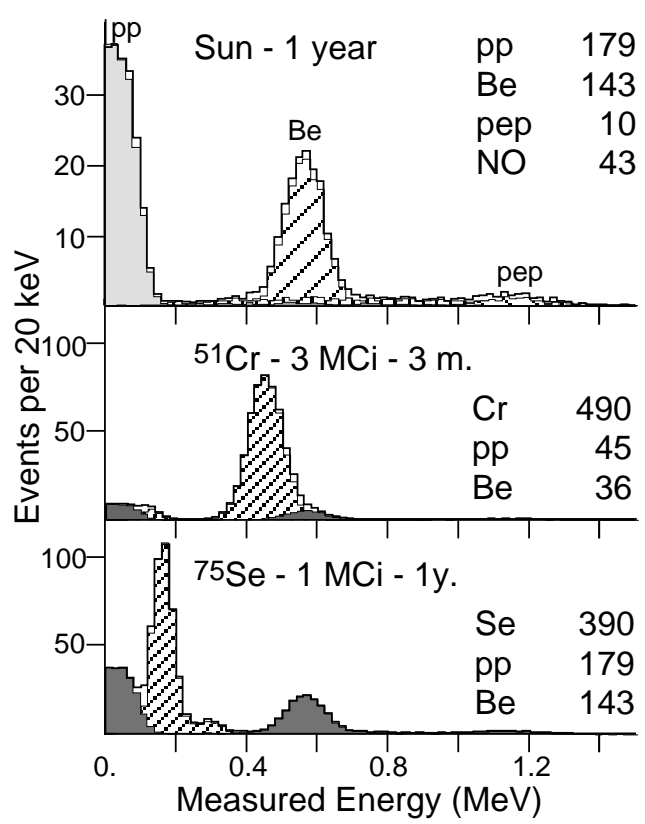

Figure 13. Estimated $\nu_{e}$ spectra of an Yb loaded liquid scintillator detector containing 20 tons of $\mathrm{Yb}$ (nat. abund.) exposed to a) solar neutrinos, b) radioactive ${ }^{51} \mathrm{Cr}$ and c) ${ }^{75} \mathrm{Se} \nu_{e}$-source [23]

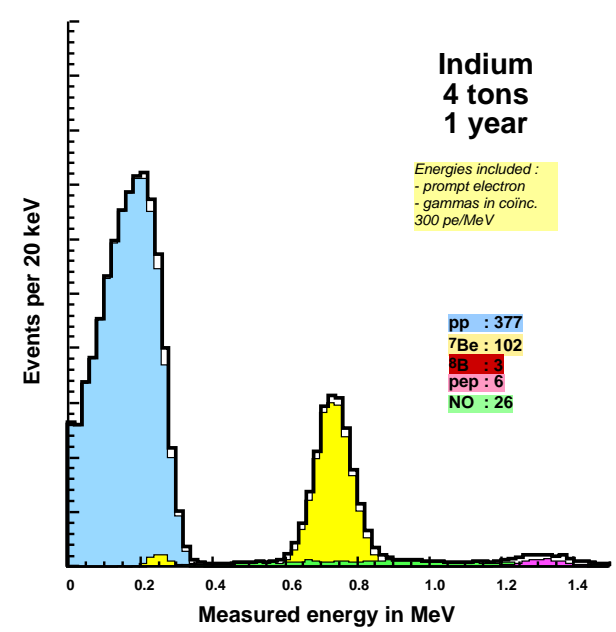

Figure 14. Estimated $\nu_{e}$ spectra of an In loaded liquid scintillator detector containing 4 tons of indium. The pp- and ${ }^{7} \mathrm{Be}$ rates are $377 /$ year and 102/year. 


\section{Study of $U_{e 3}$ with reactor- $\bar{\nu}_{e}$ 's and other projects}

The goal of the Kr2Det project [18] is the study of $U_{e 3}$ in a "disappearance" experiment using reactor- $\bar{\nu}_{e}$ 's. Two identical detectors with 50 ton mass each, similar to the design of BOREXINO, are located at $150 \mathrm{~m}$ and $1100 \mathrm{~m}$ distant from the reactor core. Both detectors will be located underground at a depth of 600 mwe. From the comparison of the event rate of both detectors, a sensitivity of $\left|U_{e 3}\right|^{2} \leq 3 \cdot 10^{-3}(90 \% \mathrm{CL})$ at $\Delta m^{2}=3 \cdot 10^{-3} \mathrm{eV}^{2}$ is expected. A prototype experiment (MiniKr2Det) with 1.5 ton target mass is under design in order to probe mixing parameters proposed by the LSND experiment.

The TEXONO collaboration in Taiwan 19] studies low-energy neutrino properties and interactions with reactor- $\bar{\nu}_{e}$ 's with HP-Ge detectors and $\mathrm{CsI}(\mathrm{Tl})$ scintillation cristalls crystals to search for a neutrino magnetic moment and neutrino decay.

\section{Summary and outlook}

Solutions with $\Delta m^{2}<10^{-6} \mathrm{eV}^{2}$ or small mixing angles can be probed best with solar neutrino experiments at sub-MeV energies. Values of $\Delta m^{2}>10^{-6} \mathrm{eV}^{2}$ and large mixing angles can be studied both with long-baseline nuclear reactor neutrino experiments and with sub-MeV solar neutrinos. Large mixing, in particular at large $\Delta m^{2}$ values, is favored when combining all solar neutrino data. In particular, the LMA solution with $\Delta m^{2}=3.7 \times 10^{-5} \mathrm{eV}^{2}$ and $\tan ^{2} \Theta=0.37$ [2] gives the best $\chi^{2}$ values in global analysis. The exact value of the minimum, as well as the extension of the allowed parameter range, depend mainly on the treatment of the ${ }^{8} \mathrm{~B}$ flux in the analysis. A large part of the $10^{-4} \mathrm{eV}^{2}$ range is allowed by the current data. Ultimately, large $\Delta m^{2}$ values are limited at $10^{-3} \mathrm{eV}^{2}$ by the $\mathrm{CHOOZ}$ reactor experiment [9].

Results of the upcoming experiments KamLAND and BOREXINO most likely will tell us which oscillation solution is realized in nature. The objective of the upcoming experiments will be to probe the mixing parameters with comple- mentary techniques both with ES and CC reactions with high precision. For the LMA solution, reactor and solar neutrino experiments are complementary to determine mixing parameters: $\Delta m^{2}$ can be derived with high precision from reactor neutrino experiments while the mixing angle is measured best with solar neutrinos. A direct measurements of the primary solar neutrino fluxes will be essential to probe, with high accuracy, solar model predictions. Perhaps even new surprises await, if so, it would not be the first time in astroparticle physics - new experiments may find new phenomena.

\section{Acknowledgement}

It is a pleasure to thank all colleagues and friends with whom I had fruitful discussions while preparing this short review of this very active and exciting field, and who provided me with the latest developments of their projects. In particular, I would like to thank F.X. Hartmann for the careful reading of the manuscript.

\section{REFERENCES}

1. T. Kirsten, Rev. Mod. Phys., 71(1999) 12131232.

2. recent golabal analysis can be found in: J.N. Bahcall, M.C. Gonzalez-Garcia, Carlos PenaGaray, hep-ph/0111150v2; P.I. Krastev and A.Yu. Smirnov, hep-ph/0108177.

3. http://www.awa.tohoku.ac.jp/KamLAND/; Proposal for US Participation in KamLAND (1999) http://kamland.lbl.gov/ KamLAND.US.Proposal.pdf.

4. BOREXINO Collaboration, G. Alimonti et al., Astrop. Phys. 16, (2002) 205-234. Also at hep-ex/0012030.

5. S. Schönert, TAUP 1997, Nucl. Phys. B (Proc. Suppl.) 70 (1999) 195-198.

6. R.S. Raghavan, PRL Vol.78 No.19 (1997) 3618; LENS collaboration, Letter of Intent, Laboratori Nazionali del Gran Sasso (1999);

7. Y. Suzuki, LowNu2 workshop, Tokyo, 2000, http://www-sk.icrr.u-tokyo.ac.jp/neutlowe/, S.Moriyama, M.Yamashita, XENON01, 
2001 ,

http://www-sk.icrr.u-

tokyo.ac.jp/xenon01/index.html

8. A. McDonald, see this proceedings; Q.R. Ahmad et al. (SNO collaboration) PRL 87 (2001) 071301.

9. M. Apollonio et al., Phys. Lett. B 466 (1999) 415.

10. J.N. Bahcall, P.I. Krastev, A.Y. Smirnov, JHEP 0105 (2001) 015

11. D.N. McKinsey and J.M. Doyle, J. of Low Temp. Phys., 118, 153 (2000).

12. B. Lanou, LowNu2 workshop, Tokyo, 2000, http://www-sk.icrr.u-tokyo.ac.jp/neutlowe/, http://www.sns.ias.edu/ jnb/Meetings/ Lownu/lownuPres/pindex.html.

13. H. Ejiri et al., PRL 85 (2000) 2917-2910

14. G. Bonvicini et al., Contributed paper, Snowmass 2001, hep-ex/0109199, hep-ex/0109032.

15. Super-MUNU, http://isnwww.in2p3.fr munu/Supermunu/solar.html; C. Broggini et al., LowNu workshop, Sudburry (2000).

16. L. Baudis, H.V. Klapdor-Kleingrothaus, Proc. Beyond the Desert'99 (1999), astroph/0003435; H.V. Klapdor-Kleingrothaus, L. Baudis, G. Heusser, B. Majorovits, H. Paes, hep-ph/9910205.

17. E. Resconi, Dissertation Univ. di Genova (2000); S. Schönert and E. Resconi, to be published; R.S. Raghavan, unpublished.

18. Y. Kozlov, L. Mikaelyan and V. Sinev, NANP-2001, Dubna (2001), hep-ph/0109277

19. C.Y. Chang et al., Nucl. Phys. B (Procs. Suppl.) 66, 419 (1998), H.T-K. Wong and J. Li, NCTS workshop 2001, hep-ex/0201001.

20. S. Schönert, T. Lasserre, L. Oberauer, to be submitted; S. Schoenert, NOON01, http://www-sk.icrr.u-tokyo.ac.jp/noon2001/

21. G. Alimonti et al., BOREXINO collaboration, Phys. Lett. B 422 (1998) 349.

22. DAMA collaboration, TAUP01, this proceedings.

23. M. Fujiwara et al., PRL 85, 4442 (2000); M.

Bhattacharya et al., PRL 85, 4446 (2000).

24. J. Rappaport et al. PRL 54, 2325 (1984).

25. R.S. Raghavan, hep-ex/0106054 (2001). 